\title{
Mining quantitative association in peptide Sequences of Flavivirus Subfamilies
}

\author{
Priyanka Rajput and Dr. Usha Chouhan \\ Department of Bioinformatics.Manit,Bhopal,India
}

\begin{abstract}
Flavivirus, one of the most important viral pathogen which is also called from the yellow fever virus(flaivirus :yellow in latin), belongs to the family Flaviviridae. The study of relationships among amino acid and other parameters in molecular sequences of these virus are crucial for understanding mechanism of pathogenicity, infection, regulation and control of the disease. In view of above an attempt has been made to develop a model for mining quantitative association patterns in the amino acid sequence of flavivirus subfamily family. The sequences have been taken form online database "NCBI". A model is propesed to generate the quantitative association relationships for 12 sub-families flavivirus. The results generated have been analyzed for similarities and differences in associations among amino-acid of this family. The association rules have been generated for redundant and non-redundant protein sequences using frequent and in-frequent patterns.

Key words:-dataset, item set, Threshold, Support, Confidence, Pattern, quantitative association mining.
\end{abstract}

\section{Introduction}

The flavivirus family contains many viral agents which produces encephalitis. Flavivirus encephalitis are either mosquito- borne, tick-borne, or have an unknown vector[4]. The viruses of the family Flaviviridae are important arthropod-borne viruses in both human and veterinary medicine. They are transmitted by mosquito and ticks and usually are maintained in a transmission cycle in nature. They are widely distributed throughout the world with the exception of the polar region, although a specific flavivirus may be geographically restricted to a continent or a particular part. They produce a broad spectrum of clinical responses in humans ranging from asymptomatic infection to fulminant encephalitis or hemorrhagic fever. Nearly 60 flaviviruses are known to exist but many are yet to be shown to cause disease in humans[5].Major symptoms include mild acute febrile syndromes, severe neurological, hepatic and/or hemorrhagic disease. The geographical diversity of flavivirus has shown the occurrence of JEV in Asia, causing menigo encephalitis in children. [6], and WNV in west Africa, middle east,and from 1999 in North America[7] .DENV shows worldwide existence , affecting 2.5 billion[8]. YFV causes serious infections manifested by fulminant hepatitis and severe hemorrhagic disease. YFV still kills a considerable number of people annually, despite the availability of an effective vaccine [9].

In 1993, Agrawal proposed an algorithm for extracting association rules from large databases [10]. Since then, association rule mining has become one of the main techniques for Knowledge Discovery in Databases (KDD).A good number of algorithms are reported in the literature [11-17] for association rule mining

Association analysis has proved to be a powerful approach for analyzing traditional market basket data, and has even been found useful for some problems in bioinformatics in a few instances. However, there are a number of other important problems in bioinformatics, such as finding biomarkers using dense data like SNP data and real-valued data like gene-expression data, where such techniques could prove to be very useful, but cannot currently be easily and effectively applied[18].

The problem of mining association rules in large relational table are introduced by Srikant $\mathrm{R}$ and agrawal [19].This technique can generate too many similar rules. They gave an algorithm for mining quantitative association rules.Attempts are also reported in the literature [21-24]for mining associations in molecular sequences.

The quantitative association rules approach read the nature of different amino acid that are present in the protein. This very basic analysis provides understandings into the Co-occurrence of certain amino acids in a protein. Such association rules are desirable for enhancing our understanding of protein composition.[20]

The peptide sequence contains lot of information about the various features and characteristics of the organism which needs to be explored by various techniques for better understanding of function and processes of the organism .In view of above a model for mining association relations in 12 sub families of flavivirus is developed . The similarity and differences in association in amino-acid of these families have been analyzed and the association rules have been generated for redundant and non-redundant protein sequences using frequent and in-frequent patterns. 


\section{Materials And Methods}

To perform the analysis of Mosquito borne the first step is to collect data of molecular sequences of sub family like :- Mosquito borne ,Tick borne and known vector from NCBI database.

For studying the frequent patterns in redundant and non-redundant sequences frequency can be calculated as

$$
\mu_{\mathrm{i}}(\mathrm{A})=\sum_{i}^{n} f_{\mathrm{i}}(\mathrm{A})
$$

where $\sum_{i}^{n} f_{\mathrm{i}}(\mathrm{A})$ is the sum of frequency of amino acid and $\mu_{\mathrm{i}}(\mathrm{A})$ is the frequency of amino acid .

$A$ is the $i^{\text {th }}$ sequence.

In this paper we assume that threshold can be defined by user

The threshold is given by

$$
\frac{\sum_{i=1}^{n} \max (a i)+\sum_{i=1}^{n} \min (a i)}{2}
$$

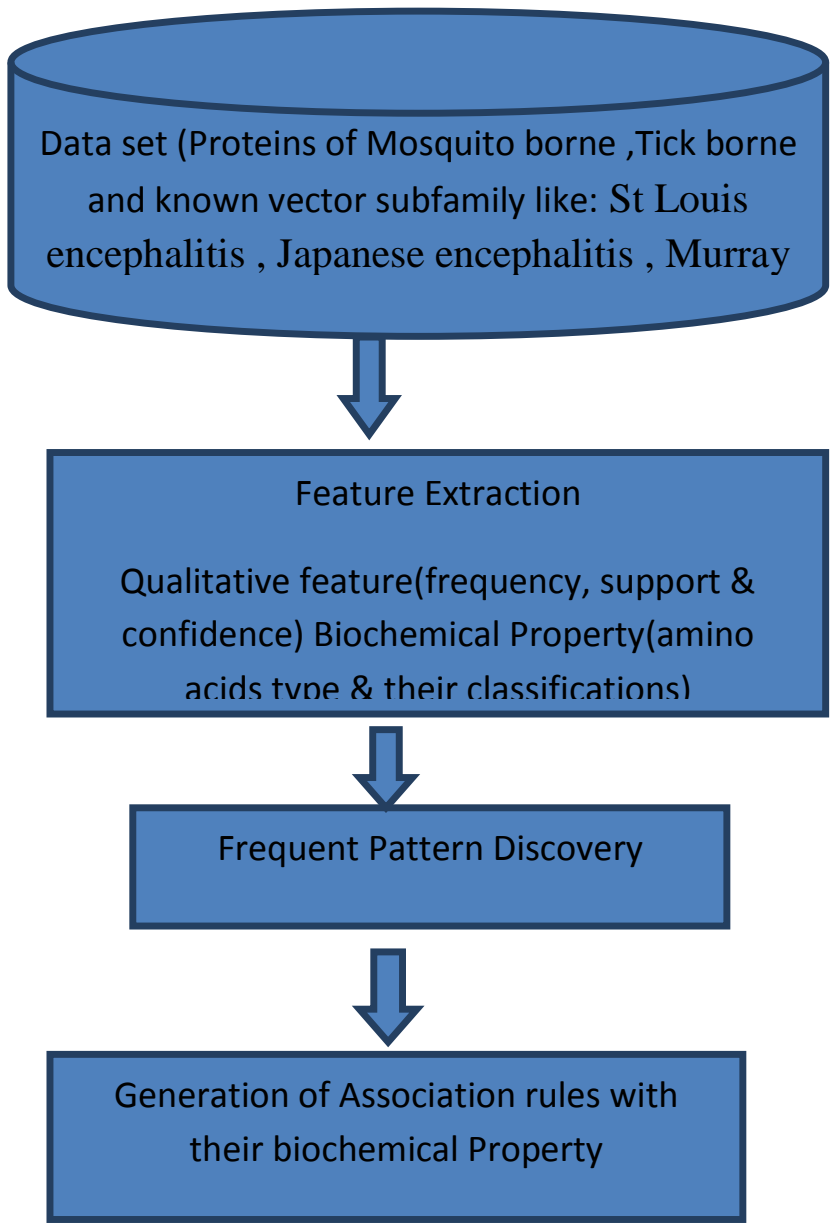

Where a is amino acid and $\mathrm{i}$ is varies from 1 to $20 \quad$ Flow chart[2]: Showing the Methodology $\mathrm{n}$ is no. of sequence.

The apriori algorithm is employed to find frequent patterns in all the sequences. These patterns are used to generate ordinary association rule.

The frequency Support for $\mathrm{n}$ amino acid can be calculated as:

$\sum \mu_{i}\left(A 1 \cap A 2 \cap A 3 \cap \ldots \ldots \ldots \ldots \ldots A_{n-1} \cap A_{n}\right)$ 


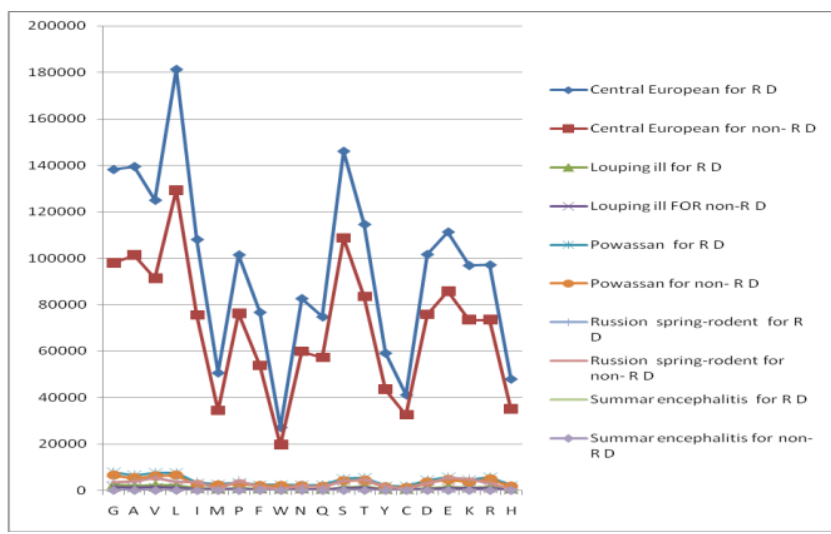

Figure2: Variation in Frequency of Redundant and Non-redundant amino acid of Tick borne subfamily

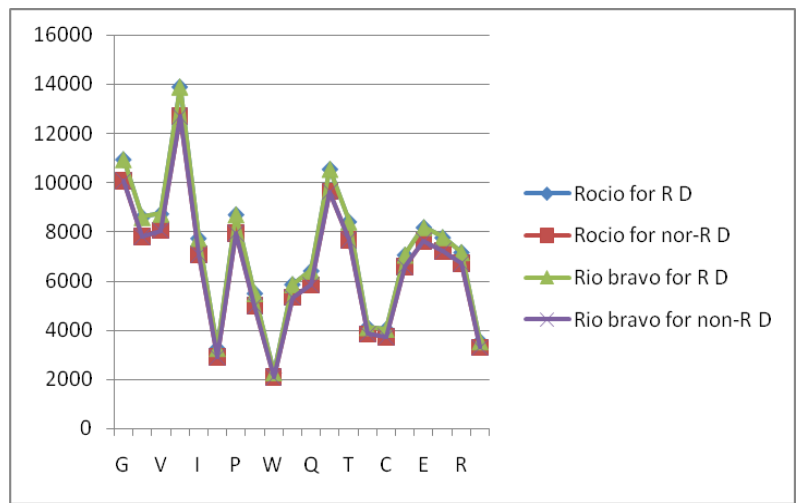

Figure3: Variation in Frequency of Redundant and Non-redundant amino acid Known Vector Subfamily

Table-1 Maximum support in case of Mosquito Borne

\begin{tabular}{|c|c|c|c|c|c|c|c|c|c|}
\hline \multicolumn{2}{|c|}{$\begin{array}{l}\text { Japanese } \\
\text { encephalitis }\end{array}$} & \multicolumn{2}{|c|}{$\begin{array}{l}\text { Marry vally } \\
\text { encephalitis }\end{array}$} & \multicolumn{2}{|c|}{ St. Louis encephalitis } & \multicolumn{2}{|l|}{ Ilheus } & \multicolumn{2}{|l|}{ West Nile } \\
\hline $\begin{array}{l}\text { Redund } \\
\text { ant }\end{array}$ & $\begin{array}{l}\text { Non- } \\
\text { redundan } \\
\mathrm{t}\end{array}$ & $\begin{array}{l}\text { Redund } \\
\text { ant }\end{array}$ & $\begin{array}{l}\text { Non- } \\
\text { redundant }\end{array}$ & $\begin{array}{l}\text { Redundan } \\
\mathrm{t}\end{array}$ & $\begin{array}{l}\text { Non- } \\
\text { redundant }\end{array}$ & Redundant & $\begin{array}{l}\text { Non- } \\
\text { redundan } \\
\mathrm{t}\end{array}$ & $\begin{array}{l}\text { Redunda } \\
\text { nt }\end{array}$ & $\begin{array}{l}\text { Non- } \\
\text { redundant }\end{array}$ \\
\hline $\begin{array}{l}\text { SUPPO } \\
\text { RT OF } \\
\text { SIX } \\
\text { FREQU } \\
\text { ENT } \\
\text { PATTE } \\
\text { RN } \\
\text { [G, A, } \\
\text { L, T, V, } \\
\text { S] }\end{array}$ & $\begin{array}{l}\text { SUPPOR } \\
\text { T OF } \\
\text { SIX } \\
\text { FREQU } \\
\text { ENT } \\
\text { PATTER } \\
\text { N } \\
\text { [E, G, A, } \\
\text { L, T, V, } \\
\text { S] }\end{array}$ & $\begin{array}{l}\text { SUPPO } \\
\text { RT OF } \\
\text { EIGHT } \\
\text { FREQ } \\
\text { UENT } \\
\text { PATTE } \\
\text { RN } \\
\text { [E, G, } \\
\text { A, L, } \\
\mathrm{K}, \mathrm{T}, \\
\mathrm{V}, \mathrm{S}]\end{array}$ & $\begin{array}{l}\text { SUPPOR } \\
\text { T OF } \\
\text { SEVEN } \\
\text { FREQUE } \\
\text { NT } \\
\text { PATTER } \\
\text { N[E, G, } \\
\text { A, L, K, } \\
\text { T, V, S, } \\
\text { R] }\end{array}$ & $\begin{array}{l}\text { SUPPOR } \\
\text { T OF } \\
\text { FIVE } \\
\text { FREQUE } \\
\text { NT } \\
\text { PATTER } \\
\text { N } \\
{[G, A, L,} \\
T, V]\end{array}$ & $\begin{array}{l}\text { SUPPORT } \\
\text { OF FIVE } \\
\text { FREQUE } \\
\text { NT } \\
\text { PATTERN } \\
{[\mathrm{G}, \mathrm{A}, \mathrm{L},} \\
\mathrm{T}, \mathrm{V}]\end{array}$ & $\begin{array}{l}\text { SUPPORT } \\
\text { OF } \\
\text { THREE } \\
\text { FREQUEN } \\
\text { T } \\
\text { PATTERN } \\
{[\mathrm{G}, \mathrm{A}, \mathrm{L}, \mathrm{I},} \\
\mathrm{T}, \mathrm{S}]\end{array}$ & $\begin{array}{l}\text { SUPPOR } \\
\text { T OF } \\
\text { THREE } \\
\text { FREQU } \\
\text { ENT } \\
\text { PATTER } \\
\text { N } \\
\text { [G, A, L, } \\
\text { I, T, V, } \\
\text { S] }\end{array}$ & $\begin{array}{l}\text { SUPPOR } \\
\text { T OF } \\
\text { SIX } \\
\text { FREQU } \\
\text { ENT } \\
\text { PATTER } \\
\text { N[E, G, } \\
\text { A, L, T, } \\
\text { V, S, R] }\end{array}$ & $\begin{array}{l}\text { SUPPOR } \\
\text { T OF } \\
\text { SEVEN } \\
\text { FREQUE } \\
\text { NT } \\
\text { PATTER } \\
\text { N } \\
\text { [E, G, A, } \\
\text { L, T, V, } \\
\text { S, R] }\end{array}$ \\
\hline $\begin{array}{l}\text { GALT } \\
\text { VS } \\
79153.0\end{array}$ & $\begin{array}{l}\text { EGALT } \\
\mathrm{V} \\
50686.0\end{array}$ & $\begin{array}{l}\text { EGAL } \\
\text { KTVS } \\
\text { SUPPO } \\
\text { RT }= \\
2490.0\end{array}$ & $\begin{array}{l}\text { EGALTV } \\
\mathrm{S} \\
\text { SUPPOR } \\
\mathrm{T}= \\
1812.0\end{array}$ & $\begin{array}{l}\text { GALTV } \\
\text { SUPPOR } \\
\mathrm{T}= \\
15208.0\end{array}$ & $\begin{array}{l}\text { GALTV } \\
\text { SUPPORT } \\
=12020.0\end{array}$ & $\begin{array}{l}\text { GAL } \\
\text { SUPPORT } \\
=3084.0\end{array}$ & $\begin{array}{l}\text { GAL } \\
\text { SUPPOR } \\
\mathrm{T}= \\
1737.0\end{array}$ & $\begin{array}{l}\text { EGALT } \\
\mathrm{V} \\
\text { SUPPOR } \\
\mathrm{T}= \\
185116.0\end{array}$ & $\begin{array}{l}\text { EGALTV } \\
\mathrm{R} \\
\text { SUPPOR } \\
\mathrm{T}= \\
123574.0\end{array}$ \\
\hline \multirow[t]{2}{*}{$\begin{array}{l}\text { TOTAL } \\
\text { NO OF } \\
\text { SIX } \\
\text { FREQU } \\
\text { ENT } \\
\text { PATTE } \\
\text { RN: } 1\end{array}$} & $\begin{array}{l}\text { GALTV } \\
\mathrm{S} \\
55074.0\end{array}$ & $\begin{array}{l}\text { TOTA } \\
\text { L NO } \\
\text { OF } \\
\text { EIGHT } \\
\text { FREQ } \\
\text { UENT } \\
\text { PATTE } \\
\text { RN: } 1\end{array}$ & $\begin{array}{l}\text { GALKTV } \\
\text { S } \\
\text { SUPPOR } \\
\text { T = } \\
1765.0\end{array}$ & $\begin{array}{l}\text { TOTAL } \\
\text { NO OF } \\
\text { FIVE } \\
\text { FREQUE } \\
\text { NT } \\
\text { PATTER } \\
\text { N: } 1\end{array}$ & $\begin{array}{l}\text { TOTAL } \\
\text { NO OF } \\
\text { FIVE } \\
\text { FREQUE } \\
\text { NT } \\
\text { PATTERN } \\
: 1\end{array}$ & $\begin{array}{l}\text { GLS } \\
\text { SUPPORT } \\
=2999.0\end{array}$ & $\begin{array}{l}\text { GLV } \\
\text { SUPPOR } \\
\mathrm{T}= \\
1582.0\end{array}$ & $\begin{array}{l}\text { GALTV } \\
\mathrm{S} \\
\text { SUPPOR } \\
\mathrm{T}= \\
195490.0\end{array}$ & $\begin{array}{l}\text { TOTAL } \\
\text { NO OF } \\
\text { SEVEN } \\
\text { FREQUE } \\
\text { NT } \\
\text { PATTER } \\
\text { N: } 1\end{array}$ \\
\hline & $\begin{array}{l}\text { TOTAL } \\
\text { NO OF } \\
\text { SIX } \\
\text { FREQU }\end{array}$ & & $\begin{array}{l}\text { TOTAL } \\
\text { NO OF } \\
\text { SEVEN } \\
\text { FREQUE }\end{array}$ & $\begin{array}{l}\text { SUPPOR } \\
\text { T OF } \\
\text { FIVE } \\
\text { FREQUE }\end{array}$ & & $\begin{array}{l}\text { ALT } \\
\text { SUPPORT } \\
=2955.0\end{array}$ & $\begin{array}{l}\text { GLS } \\
\text { SUPPOR } \\
\mathrm{T}= \\
1595.0\end{array}$ & $\begin{array}{l}\text { GALTV } \\
\mathrm{R} \\
\text { SUPPOR } \\
\mathrm{T}=\end{array}$ & \\
\hline
\end{tabular}


Mining quantitative association in peptide Sequences of Flavivirus Subfamilies

\begin{tabular}{|c|c|c|c|c|c|}
\hline $\begin{array}{l}\text { ENT } \\
\text { PATTER } \\
\mathrm{N}: 2\end{array}$ & $\begin{array}{l}\text { NT } \\
\text { PATTER } \\
\mathrm{N}: 2\end{array}$ & $\begin{array}{l}\text { NT } \\
\text { PATTER } \\
\mathrm{N} \\
{[\mathrm{G}, \mathrm{A}, \mathrm{L},} \\
\mathrm{T}, \mathrm{V}]\end{array}$ & & & 184127.0 \\
\hline & & $\begin{array}{l}\text { GALTV } \\
\text { SUPPOR } \\
\mathrm{T}= \\
15208.0\end{array}$ & $\begin{array}{l}\text { LIS } \\
\text { SUPPORT } \\
=3061.0\end{array}$ & $\begin{array}{l}\text { ALT } \\
\text { SUPPOR } \\
\mathrm{T}= \\
1586.0\end{array}$ & $\begin{array}{l}\text { TOTAL } \\
\text { NO OF } \\
\text { SIX } \\
\text { FREQU } \\
\text { ENT } \\
\text { PATTER } \\
\text { N: } 3 \\
\end{array}$ \\
\hline & & & $\begin{array}{l}\text { TOTAL } \\
\text { NO OF } \\
\text { THREE } \\
\text { FREQUEN } \\
\text { T } \\
\text { PATTERN } \\
: 4 \\
\end{array}$ & $\begin{array}{l}\text { TOTAL } \\
\text { NO OF } \\
\text { THREE } \\
\text { FREQU } \\
\text { ENT } \\
\text { PATTER } \\
\mathrm{N}: 4\end{array}$ & \\
\hline
\end{tabular}

Table-2 Maximum support in case of Tick Borne

\begin{tabular}{|c|c|c|c|c|c|c|c|c|c|}
\hline \multicolumn{2}{|c|}{ Central European } & \multicolumn{2}{|c|}{ Louping ill } & \multicolumn{2}{|l|}{ Powassan } & \multicolumn{2}{|c|}{ Russion spring-rodent } & \multicolumn{2}{|c|}{ Summar encephalitis } \\
\hline $\begin{array}{l}\text { Redunda } \\
\text { nt }\end{array}$ & $\begin{array}{l}\text { Non- } \\
\text { redundant }\end{array}$ & $\begin{array}{l}\text { Redund } \\
\text { ant }\end{array}$ & $\begin{array}{l}\text { Non- } \\
\text { redundant }\end{array}$ & Redundant & $\begin{array}{l}\text { Non- } \\
\text { redundant }\end{array}$ & Redundant & $\begin{array}{l}\text { Non- } \\
\text { redundant }\end{array}$ & $\begin{array}{l}\text { Redunda } \\
\text { nt }\end{array}$ & $\begin{array}{l}\text { Non- } \\
\text { redundant }\end{array}$ \\
\hline SUPPO & SUPPOR & SUPPO & SUPPORT & SUPPORT & SUPPORT & SUPPORT & SUPPOR & SUPPOR & SUPPORT \\
\hline RT OF & $\mathrm{TOF}$ & RT OF & OF FIVE & OF SIX & OF SIX & OF TEN & $\mathrm{T} \mathrm{OF}$ & T OF & OF FOUR \\
\hline FOUR & FOUR & FIVE & FREQUE & FREQUE & FREQUEN & FREQUEN & TEN & FOUR & FREQUE \\
\hline FREQU & FREQUE & FREQU & & & & & FREQUE & FREQUE & \\
\hline ENT & & ENT & PATTER & PATTER & PATTERN & PATTERN & NT & NT & PATTER \\
\hline PATTE & PATTER & PATTE & & & {$[\mathrm{E}, \mathrm{G}, \mathrm{A}, \mathrm{L}$, } & {$[\mathrm{E}, \mathrm{G}, \mathrm{A}, \mathrm{L}$,} & PATTER & PATTER & \\
\hline $\begin{array}{l}\text { RN } \\
{[\mathrm{G}, \mathrm{A},} \\
\mathrm{L}, \mathrm{T}, \mathrm{V}, \\
\mathrm{S}]\end{array}$ & $\begin{array}{l}\mathrm{N} \\
{[\mathrm{E}, \mathrm{G}, \mathrm{A},} \\
\mathrm{L}, \mathrm{T}, \mathrm{V}, \\
\mathrm{S}]\end{array}$ & $\begin{array}{l}\text { RN } \\
{[\mathrm{G}, \mathrm{A},} \\
\mathrm{L}, \mathrm{T}, \mathrm{V}]\end{array}$ & $\begin{array}{l}{[\mathrm{G}, \mathrm{A}, \mathrm{L},} \\
\mathrm{T}, \mathrm{V}]\end{array}$ & $\begin{array}{l}{[\mathrm{E}, \mathrm{G}, \mathrm{A},} \\
\mathrm{L}, \mathrm{T}, \mathrm{V}, \mathrm{S}, \\
\mathrm{R}]\end{array}$ & $\mathrm{T}, \mathrm{V}, \mathrm{S}, \mathrm{R}]$ & $\begin{array}{l}\mathrm{I}, \mathrm{K}, \mathrm{T}, \mathrm{V} \\
\mathrm{P}, \mathrm{S}]\end{array}$ & $\begin{array}{l}\mathrm{N} \\
{[\mathrm{E}, \mathrm{G}, \mathrm{A},} \\
\mathrm{L}, \mathrm{I}, \mathrm{K}, \mathrm{T}, \\
\mathrm{V}, \mathrm{P}, \mathrm{S}]\end{array}$ & $\begin{array}{l}\mathrm{N} \\
{[\mathrm{G}, \mathrm{A}, \mathrm{L},} \\
\mathrm{T}, \mathrm{V}]\end{array}$ & $\begin{array}{l}{[\mathrm{G}, \mathrm{A}, \mathrm{L},} \\
\mathrm{T}, \mathrm{V}]\end{array}$ \\
\hline GALV & GALV & GALTV & GALTV & EGALTV & EGALTV & EGALIKT & EGALIK & GALV & GALV \\
\hline SUPPO & SUPPOR & SUPPO & SUPPORT & SUPPORT & SUPPORT & VPS & TVPS & SUPPOR & SUPPORT \\
\hline $\begin{array}{l}\mathrm{RT}= \\
106432 . \\
0\end{array}$ & $\begin{array}{l}\mathrm{T}= \\
77644.0\end{array}$ & $\begin{array}{l}\mathrm{RT}= \\
1783.0\end{array}$ & $=1092.0$ & $=4971.0$ & $=4118.0$ & $\begin{array}{l}\text { SUPPORT } \\
=3593.0\end{array}$ & $\begin{array}{l}\text { SUPPOR } \\
\mathrm{T}= \\
3591.0\end{array}$ & $\mathrm{~T}=61.0$ & $=61.0$ \\
\hline GALS & GALS & TOTAL & TOTAL & EGALVS & EGALVS & TOTAL & TOTAL & GLTV & GLTV \\
\hline SUPPO & SUPPOR & $\mathrm{NO} O \mathrm{OF}$ & NO OF & SUPPORT & SUPPORT & NO OF & $\mathrm{NO} O \mathrm{OF}$ & SUPPOR & SUPPORT \\
\hline $\mathrm{RT}=$ & & FIVE & FIVE & $=4928.0$ & $=4097.0$ & TEN & TEN & $\mathrm{T}=59.0$ & $=59.0$ \\
\hline 105082. & 76939.0 & FREQU & FREQUE & & & FREQUEN & FREQUE & & \\
\hline & & ENT & NT & & & & NT & & \\
\hline & & $\begin{array}{l}\text { PATTE } \\
\text { RN: } 1\end{array}$ & $\begin{array}{l}\text { PATTER } \\
\mathrm{N}: 1\end{array}$ & & & $\begin{array}{l}\text { PATTERN: } \\
1\end{array}$ & $\begin{array}{l}\text { PATTER } \\
\mathrm{N}: 1\end{array}$ & & \\
\hline \multirow{9}{*}{$\begin{array}{l}\text { TOTAL } \\
\text { NO OF } \\
\text { FOUR } \\
\text { FREQU } \\
\text { ENT } \\
\text { PATTE } \\
\text { RN: } 2 \\
\end{array}$} & GLVS & & & EGALVR & EGALVR & & & TOTAL & TOTAL \\
\hline & SUPPOR & & & SUPPORT & SUPPORT & & & NO OF & $\mathrm{NO} \mathrm{OF}$ \\
\hline & $\mathrm{T}=$ & & & $=4989.0$ & $=4280.0$ & & & FOUR & FOUR \\
\hline & 75114.0 & & & & & & & FREQUE & FREQUE \\
\hline & & & & & & & & NT & \\
\hline & & & & & & & & $\begin{array}{l}\text { PATTER } \\
\mathrm{N} \cdot 2\end{array}$ & $\begin{array}{l}\text { PATTER } \\
\mathrm{N} \cdot 2\end{array}$ \\
\hline & $\begin{array}{l}\text { ALVS } \\
\text { SUPPOR } \\
\mathrm{T}= \\
76605.0\end{array}$ & & & $\begin{array}{l}\text { EGATVS } \\
\text { SUPPORT } \\
=4876.0\end{array}$ & $\begin{array}{l}\text { GALTVS } \\
\text { SUPPORT } \\
=4040.0\end{array}$ & & & & \\
\hline & $\begin{array}{l}\text { TOTAL } \\
\text { NO OF } \\
\text { FOUR } \\
\text { FREQUE } \\
\text { NT } \\
\text { PATTER } \\
\text { N: } 4\end{array}$ & & & $\begin{array}{l}\text { GALTVS } \\
\text { SUPPORT } \\
=4886.0\end{array}$ & $\begin{array}{l}\text { TOTAL } \\
\text { NO OF } \\
\text { SIX } \\
\text { FREQUEN } \\
\text { T } \\
\text { PATTERN } \\
: 4\end{array}$ & & & & \\
\hline & & & & $\begin{array}{l}\text { TOTAL } \\
\text { NO OF } \\
\text { SIX }\end{array}$ & & & & & \\
\hline
\end{tabular}




\begin{tabular}{|l|l|l|l|l|l|l|l|l|l|}
\hline & & & & FREQUE & & & & & \\
NT & & & & & \\
& & & & PATTER & & & & & \\
\hline
\end{tabular}

Table-3 Maximum support in case of Known Vector

\begin{tabular}{|c|c|c|c|}
\hline \multicolumn{2}{|l|}{ Rocio } & \multicolumn{2}{|l|}{ Rio bravo } \\
\hline Redundant & Non-redundant & Redundant & Non-redundant \\
\hline $\begin{array}{l}\text { SUPPORT OF THREE } \\
\text { FREQUENT PATTERN } \\
{[\mathrm{G}, \mathrm{A}, \mathrm{L}, \mathrm{T}, \mathrm{V}, \mathrm{P}, \mathrm{S}]}\end{array}$ & $\begin{array}{l}\text { SUPPORT OF THREE } \\
\text { FREQUENT PATTERN } \\
{[E, G, A, L, T, V, P, S]}\end{array}$ & $\begin{array}{l}\text { SUPPORT OF THREE } \\
\text { FREQUENT PATTERN } \\
{[\mathrm{G}, \mathrm{A}, \mathrm{L}, \mathrm{V}, \mathrm{S}]}\end{array}$ & $\begin{array}{l}\text { SUPPORT OF THREE } \\
\text { FREQUENT } \\
\text { PATTERN } \\
{[\mathrm{G}, \mathrm{L}, \mathrm{T}, \mathrm{V}, \mathrm{S}]}\end{array}$ \\
\hline GLT $\quad$ SUPPORT $=8132.0$ & GLT $\quad$ SUPPORT $=7407.0$ & GLV $\quad$ SUPPORT $=1344.0$ & $\begin{array}{ll}\text { GLV } & \text { SUPPORT }= \\
919.0 & \end{array}$ \\
\hline GLV $\quad$ SUPPORT $=8237.0$ & GLV $\quad$ SUPPORT $=7576.0$ & GLS $\quad$ SUPPORT $=1288.0$ & $\begin{array}{ll}\text { GLS } & \text { SUPPORT = } \\
885.0 & \\
\end{array}$ \\
\hline $\begin{array}{ll}\text { GLP } & \text { SUPPORT }=8190.0\end{array}$ & $\begin{array}{ll}\text { GLP } & \text { SUPPORT }=7511.0\end{array}$ & $\begin{array}{l}\text { TOTAL NO OF THREE } \\
\text { FREQUENT PATTERN: } 2\end{array}$ & $\begin{array}{l}\text { TOTAL NO OF } \\
\text { THREE FREQUENT } \\
\text { PATTERN: } 2\end{array}$ \\
\hline GLS $\quad$ SUPPORT $=8440.0$ & GLS $\quad$ SUPPORT $=7699.0$ & & \\
\hline $\begin{array}{l}\text { TOTAL NO OF THREE } \\
\text { FREQUENT PATTERN: } 4\end{array}$ & $\begin{array}{l}\text { TOTAL NO OF THREE } \\
\text { FREQUENT PATTERN: } 4\end{array}$ & & \\
\hline
\end{tabular}

The Tables 1,2 and 3 show maximum support for $\mathrm{k}$-frequent amino acid in case of Mosquito borne ,Tick borne and known vector sub families respectively. Based on the maximum support for frequent amino acid among the subfamilies of mosquito borne,tick borne and known vector family of Flavivirus it is found that A,G,L ,T, and V (Alanine, Glycine, Leucine, Threonine \& Valine)are frequent in all subfamilies except of Rio bravo(known vector subfamilies). S(Serine) is also frequent in all subfamilies except St. Louis encephalitis's, Louping ill and Summar encephalitis .E(Glutamate) is frequent in Japanese encephalitis(redundant dataset), st.Louis encephalitis(redundant and non-redundant dataset) and ilhues, west nile,central European(nonredundant),powassan, russion spring rodent and Rocio(non-redundant).R(Arginine) is frequent for marry vally (non-redundant dataset),west nile and powassan.K(Lysine) is frequent for Marry vally, Russion spring-roden. $\mathrm{I}($ Isoleucine) is frequent for illeus, Russion spring-rodent. $\mathrm{P}$ (Proline) is frequent for Russion spring rodent ,Rocio subfamilies of Flavi virus.

Table 4 -Probable Structures and physicochemical Prosperities of Protein Sequences of Sub families

\begin{tabular}{|c|c|c|c|c|c|c|}
\hline \multirow[t]{2}{*}{ Subfamily } & \multicolumn{3}{|l|}{ Redundant } & \multicolumn{3}{|l|}{ Non-redundant } \\
\hline & $\begin{array}{l}\text { Frequent } \\
\text { Amino Acid }\end{array}$ & $\begin{array}{l}\text { Probable } \\
\text { Structur } \\
\text { e }\end{array}$ & Physicochemical Properties & $\begin{array}{l}\text { Frequent } \\
\text { Amino Acid }\end{array}$ & $\begin{array}{l}\text { Probabl } \\
\text { e } \\
\text { Structu } \\
\text { re }\end{array}$ & Physicochemical Properties \\
\hline \multicolumn{7}{|c|}{ Mosquito Borne Subfamilies } \\
\hline $\begin{array}{l}\text { Japanese } \\
\text { encephalitis }\end{array}$ & $\begin{array}{l}\text { G, A, L, T, } \\
\text { V, S }\end{array}$ & $\begin{array}{l}\text { Helix, } \\
\text { Sheet } \\
\text { and Coil }\end{array}$ & $\begin{array}{l}\text { Polar aliphatic }(\mathrm{G}) \text {, polar } \\
\text { uncharged }(\mathrm{S}, \mathrm{T}), \text { non-polar } \\
\text { aliphatic }(\mathrm{A}, \mathrm{L}, \mathrm{V}) \\
\text { hydrophobic }(\mathrm{G}, \mathrm{L}, \mathrm{V}) \text {, } \\
\text { CBetaBranched }(\mathrm{T}, \mathrm{V})\end{array}$ & $\begin{array}{l}\text { E, G, A, L, T, } \\
V, S\end{array}$ & Helix & 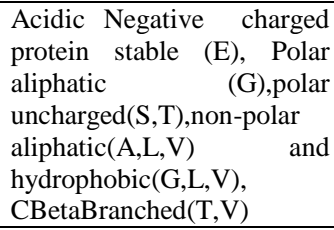 \\
\hline $\begin{array}{l}\text { Marry vally } \\
\text { encephalitis }\end{array}$ & $\begin{array}{l}\text { E, G, A, L, } \\
\text { K, T, V, S }\end{array}$ & Helix & $\begin{array}{l}\text { Acidic Negative charged } \\
\text { protein stable(E), Polar } \\
\text { aliphatic }(\mathrm{G}), \text { non-polar } \\
\text { aliphatic }(\mathrm{A}, \mathrm{L}, \mathrm{V}) \text {, and polar } \\
\text { uncharged(T,S), basic } \\
\text { charged(K) } \\
\text { hydrophobic }(\mathrm{G}, \mathrm{L}, \mathrm{V}), \\
\text { CBetaBranched(T,V) }\end{array}$ & $\begin{array}{l}\text { E, G, A, L, K, } \\
T, V, S, R\end{array}$ & Helix & $\begin{array}{l}\text { Acidic Negative charged } \\
\text { protein stable (E), Polar } \\
\text { aliphatic hydrophobic (G), } \\
\text { non-polar aliphatic }(\mathrm{A}, \mathrm{L}, \mathrm{V}) \text {, } \\
\text { polar uncharged(T,S), basic } \\
\text { charged(K,R) and } \\
\text { hydrophobic }(\mathrm{G}, \mathrm{L}, \mathrm{V}), \\
\text { CBetaBranched(T,V) }\end{array}$ \\
\hline $\begin{array}{l}\text { St. Louis } \\
\text { encephalitis }\end{array}$ & $\begin{array}{l}\text { G, A, L, T, } \\
\text { V }\end{array}$ & $\begin{array}{l}\text { Helix \& } \\
\text { Sheet }\end{array}$ & $\begin{array}{l}\text { Polar aliphatic (G),polar } \\
\text { uncharged }(\mathrm{T}) \text {,non-polar } \\
\text { aliphatic }(\mathrm{A}, \mathrm{L}, \mathrm{V}) \\
\text { hydrophobic }(\mathrm{G}, \mathrm{L}, \mathrm{V}),\end{array}$ & $\mathrm{G}, \mathrm{A}, \mathrm{L}, \mathrm{T}, \mathrm{V}$ & Helix & $\begin{array}{l}\text { Polar aliphatic (G),polar } \\
\text { uncharged( }(\mathrm{T}), \text { non-polar } \\
\text { aliphatic }(\mathrm{A}, \mathrm{L}, \mathrm{V}) \text { and } \\
\text { hydrophobic }(\mathrm{G}, \mathrm{L}, \mathrm{V}),\end{array}$ \\
\hline
\end{tabular}


Mining quantitative association in peptide Sequences of Flavivirus Subfamilies

\begin{tabular}{|c|c|c|c|c|c|c|}
\hline & & & CBetaBranched(T,V) & & & CBetaBranched(T,V) \\
\hline Ilheus & $\begin{array}{l}\text { G, A, L, I, } \\
\text { T, S }\end{array}$ & Sheet & $\begin{array}{l}\text { Polar aliphatic }(\mathrm{G}) \text {,polar } \\
\text { uncharged(S, T), non-polar } \\
\text { aliphatic }(\mathrm{A}, \mathrm{L}, \mathrm{I}) \\
\text { hydrophobic }(\mathrm{G}, \mathrm{L}) \\
\text { CBetaBranched(T) }\end{array}$ & $\begin{array}{c}\mathrm{G}, \mathrm{A}, \mathrm{L}, \mathrm{I}, \mathrm{T}, \\
\mathrm{V}, \mathrm{S}\end{array}$ & Sheet & $\begin{array}{l}\text { Polar aliphatic (G),polar } \\
\text { uncharged(S, T),non-polar } \\
\text { aliphatic }(\mathrm{A}, \mathrm{L}, \mathrm{I}, \mathrm{V}) \\
\text { hydrophobic }(\mathrm{G}, \mathrm{L}, \mathrm{V}) \text {, } \\
\text { CBetaBranched }(\mathrm{T}, \mathrm{V})\end{array}$ \\
\hline \multicolumn{7}{|c|}{ Tick Borne subfamilies } \\
\hline $\begin{array}{l}\text { Central } \\
\text { European }\end{array}$ & $\begin{array}{l}\mathrm{G}, \mathrm{A}, \mathrm{L}, \mathrm{V}, \\
\mathrm{S}\end{array}$ & $\begin{array}{l}\text { Helix } \\
\text { And coil }\end{array}$ & $\begin{array}{l}\text { Polar aliphatic (G), polar } \\
\text { uncharged(S),non-polar } \\
\text { aliphatic }(A, L, V) \\
\text { hydrophobic }(\mathrm{G}, \mathrm{L}, \mathrm{V}) \text {, } \\
\text { CBetaBranched(V) }\end{array}$ & $\begin{array}{l}\text { E, G, A, L, T, } \\
\text { V, S }\end{array}$ & $\begin{array}{l}\text { Helix, } \\
\text { Coil } \\
\text { and } \\
\text { Sheet }\end{array}$ & 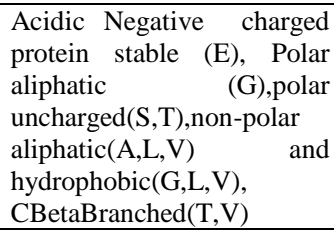 \\
\hline Louping ill & $\underset{\mathrm{V}}{\mathrm{G}, \mathrm{A}, \mathrm{L}, \mathrm{T},}$ & $\begin{array}{l}\text { Helix, } \\
\text { Coil }\end{array}$ & $\begin{array}{l}\text { Polar aliphatic }(\mathrm{G}), \text { non- } \\
\text { polar aliphatic }(\mathrm{A}, \mathrm{L}, \mathrm{V}) \text {, and } \\
\text { polar uncharged(T) and } \\
\text { hydrophobic }(\mathrm{G}, \mathrm{L}, \mathrm{V}) \text {, } \\
\text { CBetaBranched(T,V) }\end{array}$ & $\mathrm{G}, \mathrm{A}, \mathrm{L}, \mathrm{T}, \mathrm{V}$ & $\begin{array}{l}\text { Helix } \\
\text { And } \\
\text { sheet }\end{array}$ & $\begin{array}{lr}\text { Polar aliphatic hydrophobic } \\
(\mathrm{G}), \\
\text { aliphatic(A,L,V), } \\
\text { uncharged(T) } \\
\text { hydrophobic }(\mathrm{G}, \mathrm{L}, \mathrm{V}),\end{array}$ \\
\hline Powassan & $\begin{array}{l}\text { E, G, A, L, } \\
\text { T, V, S, R }\end{array}$ & $\begin{array}{l}\text { Helix \& } \\
\text { Sheet }\end{array}$ & $\begin{array}{l}\text { Acidic Negative charged } \\
\text { protein stable (E), Polar } \\
\text { aliphatic hydrophobic }(\mathrm{G}), \\
\text { non-polar aliphatic }(\mathrm{A}, \mathrm{L}, \mathrm{V}), \\
\text { polar uncharged(T,S), basic } \\
\text { charged(R) and } \\
\text { hydrophobic }(\mathrm{G}, \mathrm{L}, \mathrm{V}), \\
\text { CBetaBranched(T,V) }\end{array}$ & $\begin{array}{l}\text { E, G, A, L, T, } \\
\text { V, S, R }\end{array}$ & $\begin{array}{l}\text { Helix } \\
\& \\
\text { Sheet }\end{array}$ & $\begin{array}{l}\text { Acidic Negative charged } \\
\text { protein stable (E), Polar } \\
\text { aliphatic hydrophobic }(\mathrm{G}), \\
\text { non-polar aliphatic }(\mathrm{A}, \mathrm{L}, \mathrm{V}) \text {, } \\
\text { polar uncharged(T,S), basic } \\
\text { charged(R) and } \\
\text { hydrophobic }(\mathrm{G}, \mathrm{L}, \mathrm{V}), \\
\text { CBetaBranched(T,V) }\end{array}$ \\
\hline $\begin{array}{l}\text { Russian } \\
\text { Spring - } \\
\text { rodent }\end{array}$ & $\begin{array}{l}\mathrm{E}, \mathrm{G}, \mathrm{A}, \mathrm{L} \\
\mathrm{I}, \mathrm{K}, \mathrm{T}, \mathrm{V} \\
\mathrm{P}, \mathrm{S}]\end{array}$ & $\begin{array}{l}\text { Helix, } \\
\text { Coil } \\
\text { and } \\
\text { Sheet }\end{array}$ & $\begin{array}{l}\text { Acidic Negative charged } \\
\text { protein stable (E), Polar } \\
\text { aliphatic hydrophobic (G), } \\
\text { non-polar aliphatic(A,L,V), } \\
\text { polar ,Aliphatic Neutral } \\
\text { Non- } \\
\text { polar(P), uncharged(T,S),bas } \\
\text { ic charged(R) and } \\
\text { hydrophobic(G,L,V), } \\
\text { CBetaBranched(T,V) }\end{array}$ & $\begin{array}{l}\mathrm{E}, \mathrm{G}, \mathrm{A}, \mathrm{L}, \mathrm{I} \\
\mathrm{K}, \mathrm{T}, \mathrm{V}, \mathrm{P}, \mathrm{S}]\end{array}$ & $\begin{array}{l}\text { Helix, } \\
\text { Coil } \\
\text { and } \\
\text { Sheet }\end{array}$ & $\begin{array}{l}\text { Acidic Negative charged } \\
\text { protein stable (E), Polar } \\
\text { aliphatic hydrophobic (G), } \\
\text { non-polar aliphatic(A,L,V), } \\
\text { polar ,Aliphatic Neutral } \\
\text { Non- } \\
\text { polar(P), uncharged(T,S),bas } \\
\text { ic charged(K) and } \\
\text { hydrophobic(G,L,V), } \\
\text { CBetaBranched(T,V) }\end{array}$ \\
\hline $\begin{array}{l}\text { Summer } \\
\text { encephalitis }\end{array}$ & $\underset{V}{\mathrm{G}, \mathrm{A}, \mathrm{L}, \mathrm{T},}$ & $\begin{array}{l}\text { Helix } \\
\text { and } \\
\text { Sheet }\end{array}$ & $\begin{array}{l}\text { Polar aliphatic }(\mathrm{G}), \text { non- } \\
\text { polar aliphatic }(\mathrm{A}, \mathrm{L}, \mathrm{V}) \text {, and } \\
\text { polar uncharged(T), } \\
\text { hydrophobic }(\mathrm{G}, \mathrm{L}, \mathrm{V}), \\
\text { CBetaBranched }(\mathrm{T}, \mathrm{V}),\end{array}$ & $\mathrm{G}, \mathrm{A}, \mathrm{L}, \mathrm{T}, \mathrm{V}$ & $\begin{array}{l}\text { Helix } \\
\text { and } \\
\text { sheet }\end{array}$ & $\begin{array}{l}\text { Polar aliphatic }(\mathrm{G}), \text { non- } \\
\text { polar aliphatic }(\mathrm{A}, \mathrm{L}, \mathrm{V}) \text {, and } \\
\text { polar uncharged(T), } \\
\text { hydrophobic }(\mathrm{G}, \mathrm{L}, \mathrm{V}), \\
\text { CBetaBranched(T,V), }\end{array}$ \\
\hline \multicolumn{7}{|c|}{ Known Vector Subfamilis } \\
\hline Rocio & $\begin{array}{l}\text { G, A, L,T, } \\
\text { V,P, S }\end{array}$ & $\begin{array}{l}\text { Coil and } \\
\text { Helix }\end{array}$ & $\begin{array}{l}\text { Polar aliphatic (G), polar } \\
\text { uncharged(T,S),non-polar } \\
\text { aliphatic }(A, L, V) \text { and } \\
\text { hydrophobic }(G, L, V) \text {, Non- } \\
\text { polar(P), } \\
\text { CBetaBranched(T,V) }\end{array}$ & $\begin{array}{l}\text { E, G, A, L, T, } \\
V, P, S\end{array}$ & $\begin{array}{l}\text { Helix } \\
\text { and } \\
\text { Coil }\end{array}$ & $\begin{array}{l}\text { Acidic Negative charged } \\
\text { protein stable (E), Polar } \\
\text { aliphatic }(\mathrm{G}) \text {,polar } \\
\text { uncharged(S,T),non-polar } \\
\text { aliphatic }(\mathrm{A}, \mathrm{L}, \mathrm{V}) \text { and Non- } \\
\text { polar(P),hydrophobic(G,L,V } \\
\text { ), CBetaBranched(T,V) }\end{array}$ \\
\hline Rio bravo & $\begin{array}{l}\text { G, A, L, } \\
\text { V,S }\end{array}$ & $\begin{array}{l}\text { Helix, } \\
\text { Coil }\end{array}$ & $\begin{array}{l}\text { Polar aliphatic }(\mathrm{G}), \text { non- } \\
\text { polar aliphatic }(\mathrm{A}, \mathrm{L}, \mathrm{V}) \text {, and } \\
\text { hydrophobic }(\mathrm{G}, \mathrm{L}, \mathrm{V}), \\
\text { CBetaBranched }(\mathrm{T}, \mathrm{V})\end{array}$ & $\mathrm{G}, \mathrm{L}, \mathrm{T}, \mathrm{V}$ & Coil & $\begin{array}{lr}\text { Polar aliphatic hydrophobic } \\
(\mathrm{G}), \\
\text { aliphatic }(\mathrm{L}, \mathrm{V}), \quad \text { non-polar } \\
\text { uncharged(T) } \\
\text { hydrophobic }(\mathrm{G}, \mathrm{L}, \mathrm{V}),\end{array}$ \\
\hline
\end{tabular}

It has been found in Table 4 that maximal frequent amino acid patterns of flavi virus subfamilies are different. Most of the amino acids are common and belong to hydrophobicity, CBetaBranched, polar aliphatic and uncharged, non-polar aliphatic group. The variations among the subfamilies are in Acidic Negative Charged, Protein stability and basic positive charged group. 
Mining quantitative association in peptide Sequences of Flavivirus Subfamilies

Talbe-5 Probable Helix Structure of Protein of flavi virus based on amino acid association

\begin{tabular}{|c|c|c|c|c|c|c|c|c|}
\hline \multirow[t]{4}{*}{ Subfamily } & \multicolumn{8}{|c|}{ Helix Formation } \\
\hline & \multicolumn{8}{|c|}{$(\mathbf{A}, \mathbf{R}, \mathbf{E}, \mathbf{Q}, \mathbf{L}, \mathbf{K}, \mathbf{M}, \mathbf{H})$} \\
\hline & \multicolumn{4}{|c|}{ Redundant } & \multicolumn{4}{|c|}{ Non-redundant } \\
\hline & $\begin{array}{l}\text { 1- } \\
\text { Frequent } \\
\text { Patterns }\end{array}$ & $\begin{array}{l}\text { 2-Frequent } \\
\text { Patterns }\end{array}$ & $\begin{array}{l}\text { 3-Frequent } \\
\text { Patterns }\end{array}$ & $\begin{array}{l}\text { 4- } \\
\text { Frequent } \\
\text { Patterns }\end{array}$ & $\begin{array}{l}\text { 1- } \\
\text { Frequent } \\
\text { Patterns }\end{array}$ & $\begin{array}{l}\text { 2-Frequent } \\
\text { Patterns }\end{array}$ & $\begin{array}{l}\text { 3- } \\
\text { Frequent } \\
\text { Patterns }\end{array}$ & $\begin{array}{l}\text { 4- } \\
\text { Frequen } \\
\text { t } \\
\text { Patterns }\end{array}$ \\
\hline $\begin{array}{l}\text { Japanese } \\
\text { encephalitis }\end{array}$ & $\mathbf{A , L}$ & $\mathbf{A L}$ & None & None & $\mathbf{A , L , E , R}$ & $\begin{array}{l}\text { AL,AE,AR, } \\
\text { LE,LR }\end{array}$ & EAL,ALR & None \\
\hline $\begin{array}{l}\text { Marry vally } \\
\text { encephalitis }\end{array}$ & $\begin{array}{l}\mathbf{A}, \mathbf{L}, \mathbf{E}, \mathbf{K}, \\
\mathbf{R}\end{array}$ & $\begin{array}{l}\text { AL,AE,AK, } \\
\text { AR,LE,LK,L } \\
\text { R,EK,ER }\end{array}$ & $\begin{array}{l}\text { EAL, EAK, } \\
\text { EAR,ELK, } \\
\text { ELR,ALK, } \\
\text { ALR }\end{array}$ & EALK & $\begin{array}{l}\mathbf{A}, \mathbf{L}, \mathbf{E}, \mathbf{K}, \\
\mathbf{R}\end{array}$ & $\begin{array}{l}\text { AL,AE,AK, } \\
\text { AR,LE,LK, } \\
\text { LR,EK,ER }\end{array}$ & $\begin{array}{l}\text { EAL,EAK, } \\
\text { EAR,ELK, } \\
\text { ELR,ALK, } \\
\text { ALR }\end{array}$ & EALK \\
\hline $\begin{array}{l}\text { St. Louis } \\
\text { encephalitis }\end{array}$ & $\mathbf{A , L}$ & $\mathbf{A L}$ & None & None & $\mathbf{A , L , E , K}$ & $\mathbf{A L}$ & None & None \\
\hline Ilheus & $\mathbf{A , L}$ & $\mathbf{A L}$ & None & None & $\mathbf{A , L}$ & $\mathbf{A L}$ & None & None \\
\hline West Nile & $\begin{array}{l}\mathbf{A}, \mathbf{L}, \mathbf{E}, \mathbf{K}, \\
\mathbf{R}\end{array}$ & $\begin{array}{l}\text { AL,AE,AR, } \\
\text { LE,LR,ER }\end{array}$ & ELR,ALR & None & $\mathbf{A}, \mathbf{L}, \mathbf{E}, \mathbf{K}, \mathbf{R}$ & $\begin{array}{l}\text { AL,AE,AR, } \\
\text { LE,LK,LR, } \\
\text { ER }\end{array}$ & $\begin{array}{l}\text { EAL,ELR, } \\
\text { ALR,EAR }\end{array}$ & EALR \\
\hline $\begin{array}{l}\text { Central } \\
\text { European }\end{array}$ & $\mathbf{A}, \mathbf{L}$ & $\mathbf{A L}$ & None & None & $\mathbf{A}, \mathbf{L}, \mathbf{E}$, & AL,AE,LE & None & None \\
\hline Louping ill & $\mathbf{A , L}$ & $\mathbf{A L}$ & None & None & $\mathbf{A}, \mathbf{L}$ & $\mathbf{A L}$ & None & None \\
\hline Powassan & $\mathbf{A}, \mathbf{L}, \mathrm{E}, \mathrm{R}$ & $\begin{array}{l}\text { AL,AE,AR,LE } \\
\text {,LR,ER }\end{array}$ & $\begin{array}{l}\text { EAL,EAR } \\
\text {,ELR,ALR }\end{array}$ & EALR & $\mathbf{A}, \mathbf{L}, \mathbf{E}, \mathbf{R}$ & $\begin{array}{l}\text { AL,AE,AR } \\
\text {,LE,ER }\end{array}$ & $\begin{array}{l}\text { ALR,ELR, } \\
\text { EAL }\end{array}$ & EALR \\
\hline $\begin{array}{l}\text { Russian } \\
\text { Spring - } \\
\text { rodent }\end{array}$ & $\mathbf{A}, \mathbf{L}, \mathbf{E}, \mathbf{K}$ & AL,LE,EK,LK & $\begin{array}{l}\text { EAL,EAK } \\
\text {,ELK,ALK, }\end{array}$ & EALK & $\mathbf{A , L , E , K}$ & $\begin{array}{l}\text { AL,AE,AK, } \\
\text { EK,LK }\end{array}$ & $\begin{array}{l}\text { EAL,EAK } \\
\text {,ALK, }\end{array}$ & EALK \\
\hline $\begin{array}{l}\text { Summer } \\
\text { encephalitis }\end{array}$ & $\mathbf{A}, \mathbf{L}$, & $\mathbf{A L}$ & None & None & $\mathbf{A , L}$ & $\mathbf{A L}$ & None & None \\
\hline Rocio & $\mathbf{A}, \mathbf{L}$ & $\mathbf{A L}$ & None & None & $\mathbf{A , E , L}$ & $\mathbf{A L}, \mathbf{E L}$ & None & None \\
\hline Rio bravo & $\mathbf{A}, \mathbf{L}$ & $\mathbf{A L}$ & None & None & $\mathbf{L}$ & None & None & None \\
\hline
\end{tabular}

Talbe-6 Probable Sheet Structure of Protein of flavivirus based on amino acid association

\begin{tabular}{|c|c|c|c|c|c|c|c|c|}
\hline \multirow{4}{*}{ Subfamily } & \multicolumn{8}{|c|}{ Sheet Formation } \\
\hline & \multicolumn{8}{|c|}{$(\mathrm{V}, \mathrm{I}, \mathrm{T}, \mathrm{C}, \mathrm{W}, \mathrm{F}, \mathrm{Y})$} \\
\hline & \multicolumn{4}{|c|}{ Redundant } & \multicolumn{4}{|c|}{ Non-redundant } \\
\hline & $\begin{array}{l}\text { 1-Frequent } \\
\text { Patterns }\end{array}$ & $\begin{array}{l}\text { 2-Frequent } \\
\text { Patterns }\end{array}$ & $\begin{array}{l}\text { 3- } \\
\text { Frequent } \\
\text { Patterns }\end{array}$ & $\begin{array}{l}\text { 4- } \\
\text { Frequen } \\
\text { t } \\
\text { Patterns } \\
\end{array}$ & $\begin{array}{l}\text { 1-Frequent } \\
\text { Patterns }\end{array}$ & $\begin{array}{l}\text { 2-Frequent } \\
\text { Patterns }\end{array}$ & $\begin{array}{l}\text { 3- } \\
\text { Frequent } \\
\text { Patterns }\end{array}$ & $\begin{array}{l}\text { 4- } \\
\text { Frequen } \\
\text { t } \\
\text { Patterns } \\
\end{array}$ \\
\hline $\begin{array}{l}\text { Japanese } \\
\text { encephalitis } \\
\text { Threshold } \\
\mathbf{1 2 1 1 2 . 5}\end{array}$ & $\mathbf{V , T}$ & VT & None & None & $\mathbf{V , T}$ & VT & None & None \\
\hline $\begin{array}{l}\text { Marry } \\
\text { vally } \\
\text { encephalitis }\end{array}$ & $\mathbf{V}, \mathbf{T}$ & VT & None & None & $\mathbf{V}, \mathbf{T}$ & VT & None & None \\
\hline $\begin{array}{l}\text { St. Louis } \\
\text { encephalitis }\end{array}$ & $\mathbf{V}, \mathbf{T}$ & VT & None & None & $\mathbf{V}, \mathbf{T}$ & VT & None & None \\
\hline Ilheus & $\mathbf{V , I , T}$ & None & None & None & $\mathbf{V , I , T}$ & None & None & None \\
\hline West Nile & $\mathbf{V}, \mathbf{T}$ & VT & None & None & $\mathbf{V}, \mathbf{T}$ & VT & None & None \\
\hline $\begin{array}{l}\text { Central } \\
\text { European }\end{array}$ & $\mathbf{V}, \mathbf{T}$ & None & None & None & $\mathbf{V}, \mathbf{T}$ & VT & None & None \\
\hline Louping ill & $\mathbf{V}, \mathbf{T}$ & VT & None & None & $\mathbf{V}, \mathbf{T}$ & VT & None & None \\
\hline Powassan & $\mathbf{V}, \mathbf{T}$ & VT & None & None & $\mathbf{V}, \mathbf{T}$ & VT & None & None \\
\hline
\end{tabular}


Mining quantitative association in peptide Sequences of Flavivirus Subfamilies

\begin{tabular}{|l|l|l|l|l|l|l|l|l|}
\hline & & & & & & & & \\
\hline $\begin{array}{l}\text { Russian } \\
\text { Spring - } \\
\text { rodent }\end{array}$ & $\mathbf{V}, \mathbf{I , T}$ & VL,VI & LIV & None & V,I,T & VI,VL,LI & ITV & None \\
\hline $\begin{array}{l}\text { Summer } \\
\text { encephalitis }\end{array}$ & $\mathbf{V}, \mathbf{T}$ & $\mathbf{V T}$ & None & None & $\mathbf{V}, \mathbf{T}$ & VT & None & None \\
\hline Rocio & $\mathbf{V}, \mathbf{T}$ & None & None & None & $\mathbf{V , T}$ & None & None & None \\
\hline Rio bravo & $\mathbf{V}$ & None & None & None & $\mathbf{V , T}$ & None & None & None \\
\hline
\end{tabular}

Talbe-7 Probable Coil Structure of Protein of flavivirus based on amino acid association

\begin{tabular}{|c|c|c|c|c|c|c|c|c|}
\hline \multirow[t]{4}{*}{ Subfamily } & \multicolumn{8}{|l|}{ Coil } \\
\hline & \multicolumn{8}{|l|}{$(\mathrm{N}, \mathrm{D}, \mathrm{P}, \mathrm{S}, \mathrm{G})$} \\
\hline & \multicolumn{4}{|l|}{ Redundant } & \multicolumn{4}{|c|}{ Non-redundant } \\
\hline & $\begin{array}{l}\text { 1-Frequent } \\
\text { Patterns }\end{array}$ & $\begin{array}{l}\text { 2-Frequent } \\
\text { Patterns }\end{array}$ & $\begin{array}{l}3- \\
\text { Frequent } \\
\text { Patterns }\end{array}$ & $\begin{array}{l}\text { 4-Frequent } \\
\text { Patterns }\end{array}$ & $\begin{array}{l}\text { 1-Frequent } \\
\text { Patterns }\end{array}$ & $\begin{array}{l}\text { 2-Frequent } \\
\text { Patterns }\end{array}$ & $\begin{array}{l}\text { 3-Frequent } \\
\text { Patterns }\end{array}$ & $\begin{array}{l}\text { 4- } \\
\text { Frequ } \\
\text { ent } \\
\text { Patter } \\
\text { ns }\end{array}$ \\
\hline $\begin{array}{l}\text { Japanese } \\
\text { encephalitis } \\
\text { Threshold } \\
12112.5\end{array}$ & $\mathbf{G , S}$ & GS & none & None & G,S & GS & None & None \\
\hline $\begin{array}{l}\text { Marry vally } \\
\text { encephalitis }\end{array}$ & $\mathbf{G , S}$ & GS & None & None & $\mathbf{G , S}$ & GS & None & None \\
\hline $\begin{array}{l}\text { St. Louis } \\
\text { encephalitis }\end{array}$ & G & None & None & None & G & None & None & None \\
\hline Ilheus & G,S & GS & None & None & G,S & GS & None & None \\
\hline West Nile & G,S & GS & None & None & G,S & SG & None & None \\
\hline $\begin{array}{l}\text { Central } \\
\text { European }\end{array}$ & G,S & GS & none & None & G & None & None & None \\
\hline Louping ill & G & None & None & None & G & None & None & None \\
\hline Powassan & $\begin{array}{l}\mathbf{G}, \\
\mathbf{S}\end{array}$ & GS & None & None & $\mathbf{G , S}$ & GS & None & None \\
\hline $\begin{array}{l}\text { Russian } \\
\text { Spring - } \\
\text { rodent }\end{array}$ & G,S,P & GS,GP,PS & GPS & None & G,S,P & GS,GP,PS & GPS & None \\
\hline $\begin{array}{l}\text { Summer } \\
\text { encephalitis }\end{array}$ & G & None & None & None & $\mathbf{G}$ & None & None & None \\
\hline Rocio & $\mathbf{G}, \mathbf{P}, \mathbf{S}$ & GP,GS & none & None & $\mathbf{G}, \mathrm{P}, \mathrm{S}$ & GP,GS & None & None \\
\hline Rio bravo & $\mathbf{G , S}$ & GS & None & none & $\begin{array}{l}\mathbf{G}, \\
\mathbf{S}\end{array}$ & GS & None & None \\
\hline
\end{tabular}

The Table 5 presents the amino acid association patterns which are responsible for formation of secondary structure Helix, in various Flavi virus subfamilies. It has been observed that redundant dataset of Japanese encephalitis (Mosqiti borne subfamily),Central European (Tick borne subfamily) and Rocio (Known Vector) have two frequent patterns AL (amino acid Alanine and Lucien) which are responsible for formation of Helix and non-redundant dataset of Japanese encephalitis (Mosqiti borne subfamily),Central European (Tick borne subfamily) and Rocio(Known Vector) have three frequent patterns A, L, E (amino acid Alanine, Lucien and Glutamic acid) which are responsible for formation of secondary structure Helix. Along with Japanese encephalitis has amino acid R(Arginine) which are responsible for formation of Helix. Table 6 and 7 presents the amino acid association patterns which are responsible for formation of secondary structure Sheet and Coil respectively in various flavi virus sub families .

In Table 6 it has been observed that redundant and non- redundant dataset of Japanese encephalitis (Mosqiti borne subfamily),Central European (Tick borne subfamily) and Rocio (Known Vector) have two frequent patterns VT (amino acid Valine and Threonine) which are responsible for formation of Sheet.

In Table 7 it's observed that most of subfamilies have two frequent patterns GS which are responsible for formation of secondary structure Coil.and amino acid $\mathrm{P}$ (proline) is also present in the some subfamilies which is 
important in coil formation. Parallel analysis can be ready for rest of the subfamilies in table 5,6 and 7 to produce association rule.

It has been shown in table 5,6 and 7 that in all the 12 subfamilies of Flavi virus family, association patterns of amino acid exposed high tendency to form Helix rather than Sheet and Coil.

The association rules generated on the basis of aboveFlavi Virus Subfamilies are given below:-

Mosquito Borne Subfamilies:-

For 2 frequent Patterns:-

I. $\quad\{\mathbf{A}($ Frequent $) \cap \mathbf{L}($ Frequent $)=>$ Tendency for Helix Formation $\}$

II. $\quad\{\mathrm{A}($ Frequent $) \cap \mathrm{E}($ Frequent $)=>$ Tendency for Helix Formation $\}$

III. $\{A($ Frequent $) \cap K($ Frequent $)=>$ Tendency for Helix Formation $\}$

IV. $\quad\{\mathbf{A}($ Frequent $) \cap \mathbf{R}($ Frequent $)=>$ Tendency for Helix Formation $\}$

V. $\{\mathbf{L}($ Frequent $) \cap \mathbf{E}($ Frequent $)=>$ Tendency for Helix Formation $\}$

VI. $\quad\{L($ Frequent $) \cap K($ Frequent $)=>$ Tendency for Helix Formation $\}$

VII. $\{\mathbf{L}($ Frequent $) \cap \mathbf{R}($ Frequent $)=>$ maintain charge of protein and help in protein stability $\}$

VIII. $\{$ E(Frequent) $\cap K$ (Frequent $)=>$ Tendency for Helix Formation and Protein Solubility $\}$

IX. $\quad\{$ E(Frequent $) \cap R($ Frequent $)=>$ Tendency for Helix Formation and Protein Solubility $\}$

X. $\quad\{$ V(Frequent $) \cap T($ Frequent $)=>$ Tendency for Sheet Formation $\}$

XI. $\{\mathbf{G}($ Frequent $) \cap S($ Frequent $)=>$ Tendency for Coil Formation $\}$

For 3 frequent Patterns:-

I. $\quad\{\mathbf{E}($ Frequent $) \cap A($ Frequent $) \cap L($ Frequent $)=>$ Tendency for Helix Formation $\}$

II. $\quad\{A($ Frequent $) \cap L($ Frequent $) \cap R($ Frequent $)=>$ Tendency for Helix Formation $\}$

III. $\{\mathbf{E}($ Frequent $) \cap \mathbf{A}($ Frequent $) \cap K($ Frequent $)=>$ Tendency for Helix Formation $\}$

IV. $\{\mathbf{E}($ Frequent $) \cap \mathbf{A}($ Frequent $) \cap R($ Frequent $)=>$ Tendency for Helix Formation $\}$

V. $\{\mathbf{E}($ Frequent $) \cap L($ Frequent $) \cap K($ Frequent $)=>$ Tendency for Helix Formation $\}$

VI. $\quad\{$ A(Frequent $) \cap L($ Frequent $) \cap K($ Frequent $)=>$ Tendency for Helix Formation $\}$

VII. $\{$ E(Frequent $) \cap L($ Frequent $) \cap R($ Frequent $)=>$ Tendency for Helix Formation\}

For 4 frequent Patterns:-

I. $\{\mathbf{E}($ Frequent $) \cap \mathbf{A}($ Frequent $) \cap \mathbf{L}($ Frequent $) \cap K($ Frequent $)=>$ Tendency for Helix Formation $\}$

II. $\{$ E(Frequent $) \cap A($ Frequent $) \cap L($ Frequent $) \cap R($ Frequent $)=>$ Tendency for Helix Formation $\}$

For Tick Borne Subfamilies:-

For 2 Frequent Patterns

$\{A($ Frequent $) \cap L($ Frequent $)=>$ Tendency for Helix Formation $\}$

I. $\quad\{\mathbf{A}($ Frequent $) \cap E($ Frequent $)=>$ Tendency for Helix Formation $\}$

II. $\{\mathbf{A}($ Frequent $) \cap \mathbf{R}($ Frequent $)=>$ Tendency for Helix Formation $\}$

III. $\{$ L(Frequent $) \cap E($ Frequent $)=>$ Tendency for Helix Formation $\}$

IV. $\{L($ Frequent $) \cap K($ Frequent $)=>$ Tendency for Helix Formation $\}$

V. $\{\mathbf{L}($ Frequent $) \cap R($ Frequent $)=>$ maintain charge of protein and help in protein stability

VI. $\{\mathbf{E}($ Frequent $) \cap K($ Frequent $)=>$ Tendency for Helix Formation and Protein Solubility $\}$

VII. $\quad\{$ E (Frequent $) \cap R($ Frequent $)=>$ Tendency for Helix Formation and Protein Solubility $\}$

VIII. $\{$ V(Frequent $) \cap T($ Frequent $)=>$ Tendency for Sheet Formation $\}$

IX. $\quad\{$ V(Frequent $) \cap I($ Frequent $)=>$ Tendency for Sheet Formation $\}$

X. $\{$ L(Frequent $) \cap($ Frequent $)=>$ Tendency for Sheet Formation $\}$

XI. $\{\mathbf{G}($ Frequent $) \cap S($ Frequent $)=>$ Tendency for Coil Formation $\}$

XII. $\quad\{G($ Frequent $) \cap P($ Frequent $)=>$ Tendency for Coil Formation\}

XIII. $\quad\{P($ Frequent $) \cap S($ Frequent $)=>$ Tendency for Coil Formation $\}$

For 3 frequent Patterns:-

VIII. $\{$ E(Frequent $) \cap A($ Frequent $) \cap L($ Frequent $)=>$ Tendency for Helix Formation $\}$

IX. $\quad\{$ A(Frequent $) \cap L($ Frequent $) \cap R($ Frequent $)=>$ Tendency for Helix Formation

X. $\{$ E(Frequent $) \cap A($ Frequent $) \cap K($ Frequent $)=>$ Tendency for Helix Formation $\}$

XI. $\{\mathbf{E}($ Frequent $) \cap A($ Frequent $) \cap R($ Frequent $)=>$ Tendency for Helix Formation $\}$

XII. $\quad$ E(Frequent $) \cap L($ Frequent $) \cap K($ Frequent $)=>$ Tendency for Helix Formation $\}$

XIII. \{ A(Frequent $) \cap L($ Frequent $) \cap K($ Frequent) $=>$ Tendency for Helix Formation $\}$

XIV. $\{$ E(Frequent $) \cap L($ Frequent $) \cap R($ Frequent $)=>$ Tendency for Helix Formation $\}$

XV. $\{$ I (Frequent $) \cap T($ Frequent $) \cap V($ Frequent $)=>$ Tendency for Sheet Formation $\}$

XVI. $\{G($ Frequent $) \cap P($ Frequent $) \cap S($ Frequent $)=>$ Tendency for Coil Formation

For 4 frequent Patterns:-

I. I. $\{\mathbf{E}$ (Frequent $) \cap A($ Frequent $) \cap L($ Frequent $) \cap K($ Frequent $)=>$ Tendency for Helix Formation $\}$

II. $\{\mathbf{E}($ Frequent $) \cap A($ Frequent $) \cap L($ Frequent $) \cap R($ Frequent $)=>$ Tendency for Helix Formation $\}$

For Known Vector:-

For 2 Frequent Patterns:-

I. $\quad\{$ A(Frequent $) \cap L($ Frequent $)=>$ Tendency for Helix Formation $\}$

II. $\{\mathbf{G}($ Frequent $) \cap S($ Frequent $)=>$ Tendency for Coil Formation $\}$

III. $\{G($ Frequent $) \cap P($ Frequent $)=>$ Tendency for Coil Formation $\}$ 


\section{Conclusion}

The approach for mining quantitative associations is appropriate in view of analysis and prediction of secondary structure of sequences for each subfamily of flavivirus. Further quantitative associations have been obtained successfully to generate association rules for predicting the physiochemical properties and secondary structure. This approach will lead to develop fuzzy set and soft set based approaches for mining association in flavivirus sequences and their structures. These studies can be performed to generate association rules, signatures and their relationships along with physiochemical properties.

\section{Conflict Of Interest}

The authors are no conflict of interest.

\section{ACKNOWLEDGMENT}

The authors are grateful to Department of Biotechnology, New Delhi for Providing Bioinformatics Infrastructure facility at MANIT Bhopal to carry out this.

\section{References}

[1]. M. W. Gaunt, A. A. Sall, X. de Lamballerie, A. K. I. Falconar, T. I. Dzhivanian, and E. A. Goul(2001)d, "Phylogenetic relationships of flaviviruses correlate with their epidemiology, disease association and biogeography," Journal of General Virology, vol. 82, part 8, pp. 1867-1876.

[2]. Shi, P-Y (editor) (2012). Molecular Virology and Control of Flaviviruses. Caister Academic Press. ISBN 978-1-904455-92-9.

[3]. A. Oya and I. Kurane(2007), "Japanese encephalitis for a reference to international travelers," Journal of Travel Medicine, vol. 14, no. 4, pp. 259-268.

[4]. PanisadeeAvirutnan,Anja Fuchs, Richard E. Hauhart( March 2010),"Antagonism of the complement component C4 by flavivirusnonstructural protein NS1"J Exp Med 2010 207:793-806

[5]. B. J. Blitvich(2008), "Transmission dynamics and changing epidemiology of West Nile virus," Animal Health Research Reviews, vol. 9 , no. 1 , pp. $71-86$.

[6]. S. B. Halstead(2007), "Dengue," The Lancet, vol. 370, no. 9599, pp. 1644-1652.

[7]. E. Gould and T. Solomon(2008), "Pathogenic flaviviruses," The Lancet, vol. 371, no. 9611, pp. 500-509.

[8]. Jaiwei Han and Yongjian (1995)"Discovery of multiple level association rule from large database",VLDB Conference, pp. 420-431.

[9]. PanisadeeAvirutnan,Anja Fuchs, Richard E. Hauhart( March 2010), "Antagonism of the complement component C4 by flavivirusnonstructural protein NS1'J Exp Med 2010 207:793-806

[10]. Gowtham Atluri at al Association Analysis Techniques for Bioinformatics Problems" \{gowtham,rohit,gangfang,gaurav,steinbac,kumar\}@cs.umn.edu,http://www.cs.umn.edu/ kumar/dmbio

[11]. Pandey, Anjana and KamalRaj Pardasani.’Rough set Model for Discovering Multidimensional Association Rules.'International Journal of Computer Science and Network Security9.6(2009):159-164.

[12]. Khare,Neelu,Neeru Adlakha, and K.R. Pardasani."An Algorithm for Mining Multidimensional Association Rules using Boolean Matix.'Recent Trends in Information,Telecommunication and Computing (ITC),2010 International Conference on.IEEE,2010.

[13]. Khare,N.;Adlakha,N.;Pardasani,K.R.”An algorithm for mining conditional hybrid dimensional association rule using Boolean Matix,"Computer and automation engineering (ICCAE),2010 The $2^{\text {nd }}$ International Conference on,vol.2,no.,pp.644,648,26-28 Feb.2010

[14]. Khare ,Neelu Adlakha, and K.R. Pardasani.’Karnaugh MAP Model for Mining Association Rules in Large Databases.”(IJCNS) International Journal of Computer and Network Security 1.1(2009).

[15]. Pandey,Anjana,Niket Bhargava, and K.R. Pardasani."Counting inference appoarch to discover calendar based temporal association rules." SPIT-IEEE Colloquium. Vol.5.2007.

[16]. Panday,Anjana, and K.R. Pardasani."PPCI algorithm for mining temporal association rules in large database.”Journal of Information \& Knowledge Management 8.04(2009):345-352.

[17]. Khare,Neelu,Neelu Adlakha, and K.R. Pardasani.”An Algorithm for Mining Multidimensional Fuzzy Association Rules.”arXiv preprint arXiv:0909.5166(2009).

[18]. Agrawal,R. and Srikant,R.(1994).Fast algorithms for mining association rules .In proc of the $20^{\text {th }}$ Int'l Conference on Very Large Databases,407-419

[19]. Srikant R and Agrawal(1996) "Mining quantitative association rules in large relational tables" SIGMOD '96 Proceedings of the 1996 ACM SIGMOD international conference on Management of data, Volume 25 Issue 2, June 1996 Pages 1-12

[20]. Nitin Gupta, Nitin Mangal, Kamal Tiwari, Pabitra Mitra(2006) "Mining Quantitative Association Rules in Protein Sequences" Lecture Notes in Computer Science Volume 3755, pp 273-281

[21]. Anuja Shankar \& KamalRAj Pardasani”Mining Fuzzy amino acid association patterns in various orders of class Apphaproteobacteria”Journal of Medical Imaging and Health Informatics.Accepted for Publication 2013

[22]. Kumari T,Pardasani KR.,'Mining Fuzzy amino acid Association Patterns in Class C GPCRs"Computational Life sciences,Springer.Accepted of Publication 2013

[23]. Kumari T, Pardasani KR.,'Mining Fuzzy associations among amino acids of class A GPCRs.”OnlineJ Bioinform.,13(2):202213,2012

[24]. Anuja Shankar \& Kamal Raj Pardasani,"Amino acid composition based model for prediction and identification of Alpa and Epsilon-proteobacteria"Online Journal of Bioinformatics,14(1)(2013)

Publiched date 14 mar 2014 